\title{
COVID-19 clinical trials: learning from exceptions in the research chaos
}

To the Editor - Jeremy Farrar, Director of Wellcome and Chair of the World Health Organization R\&D Blueprint Scientific Advisory Group, has said "It's critical that the global research effort is rapid, robust and is conducted at scale and co-ordinated across multiple countries."

Of the $>2,000$ planned drug studies examining COVID-19 treatments (https:// www.covid-trials.org), most have delivered little or no directly useful information ${ }^{1}$. Exceptions include two large, adaptive, pragmatic trials, RECOVERY and SOLIDARITY, which combined have randomized $>20,000$ patients to assess the effects of several treatments on mortality ${ }^{2}$, and the US National Institutes of Health ACTT trial, which randomized 1,059 patients to assess the effect of remdesivir on time to disease resolution ${ }^{3}$.

The speed of the RECOVERY trial (https://www.recoverytrial.net/files/ recovery-protocol-v7-0-2020-06-18.pdf) was record-breaking: the period from protocol to first patient recruitment was nine days, with the 176 UK hospitals recruiting $>12,000$ hospitalized patients (15\% UK COVID-19 cases), and it provided clear answers within a few months on the effectiveness of dexamethasone $e^{2}$ and the ineffectiveness of hydroxychloroquine and lopinavirritonavir (Fig. 1). Notably, it sought to achieve reliability and quality by design rather than by compliance with good clinical practice or site monitors, relying instead on centralized computer checks on site behavior and patient compliance, and utilizing central National Health Service medical records of treatment and outcome. Also, it did not necessarily require written consent where the medical emergency rendered this inappropriate.

The World Health Organization-led SOLIDARITY trial (https://www.who.int/ emergencies/diseases/novel-coronavirus2019/global-research-on-novel-coronavirus2019-ncov/solidarity-clinical-trial-for-covid19-treatments) has recruited $>9,000$ patients across 30 countries and is currently recruiting about 2,000 patients a month. SOLIDARITY has also provided important evidence on the ineffectiveness of hydroxychloroquine and lopinavirritonavir and is the largest trial examining the effects of remdesivir and interferon on

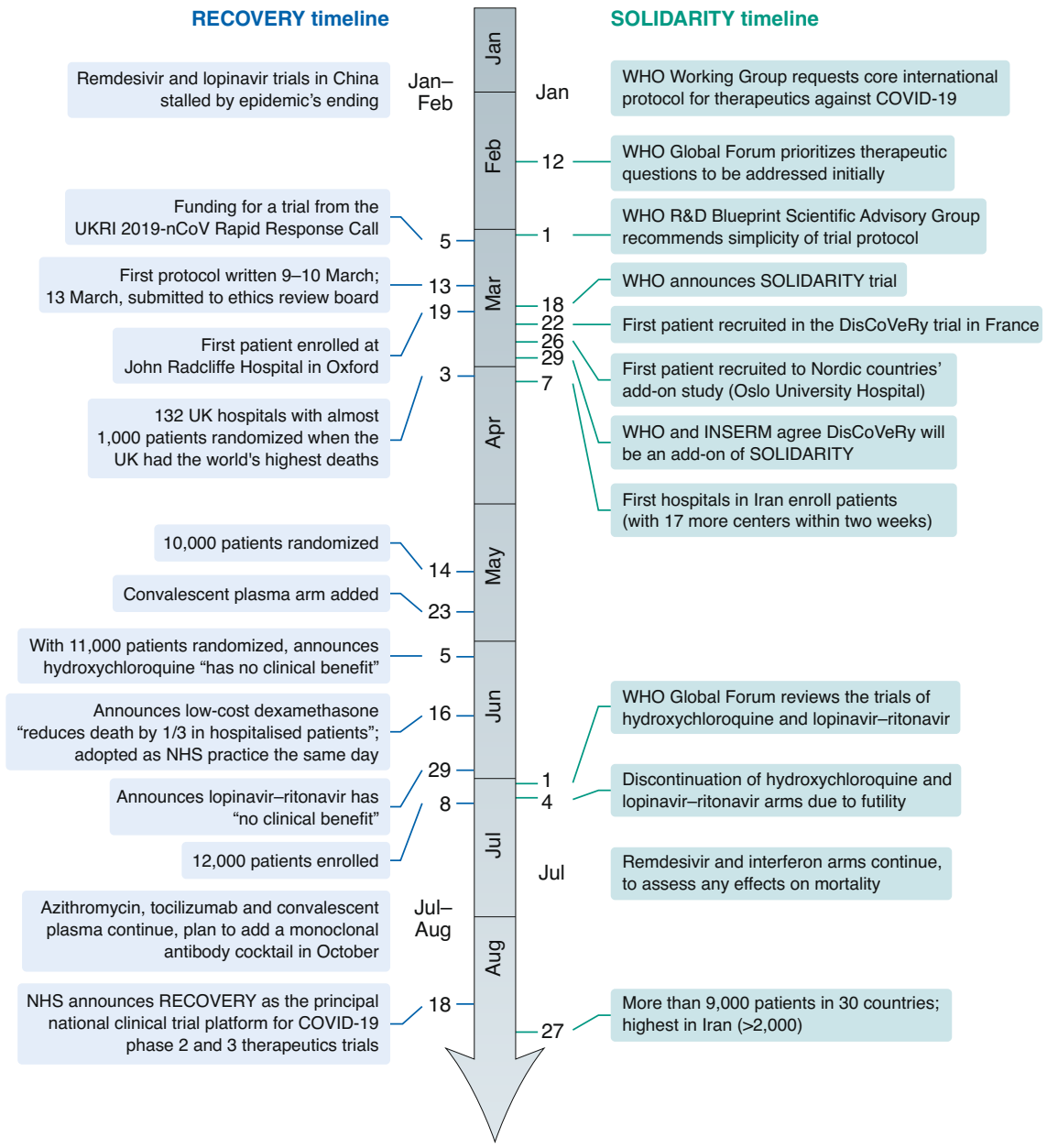

Fig. 1 | RECOVERY and SOLIDARITY timelines. NHS, National Health Service; WHO, World Health Organization.

survival. SOLIDARITY also includes add-on sub-studies (Nordic and Canadian arms and the European DisCoVeRy trial; https:// presse.inserm.fr/en/launch-of-a-europeanclinical-trial-against-covid-19/38737/) that provide more-detailed data on safety and clinical and laboratory surrogates.

Both trials have common origins in the international collaborative work enabled by the World Health Organization R\&D Blueprint (Fig. 1). The RECOVERY protocol, written in only two days, was heavily influenced by the 1980s ideas of large, simple, pragmatic trials ${ }^{4}$, exemplified by the International Studies of Infarct Survival ${ }^{5}$ : broad, simple inclusion criteria; central randomization; no additional biological samples or extraneous data collection; and the simple, unambiguous primary outcome of all-cause mortality. RECOVERY benefited from the willingness of UK doctors to randomize unproven treatments (rather than, as in many other countries, obstructing randomization by incorporating unproven drugs into local practices and guidelines); strong national support from funders and the National Health Service; and the pre-existing UK clinical-trials network, which provided research nurses at recruiting centers ${ }^{6}$. RECOVERY's streamlined trial conduct and data collection ${ }^{7}$ - including foregoing 
formulaic training on good clinical practice - enabled the participation of hospitals already stretched by patients with COVID-19, with many of the less-research-experienced hospitals being among the best recruiters. Finally, a strong letter of support from the Chief Medical Officers of England, Wales, Scotland and Northern Ireland emphasized that the trial was to be seen as part of clinical care and stated that "Use of treatments outside of a trial, where participation was possible, is a wasted opportunity to create information that will benefit others."

The SOLIDARITY protocol was also a simple, pragmatic design adapted rapidly from a pre-existing core pandemic protocol. Multi-country involvement allowed recruitment to shift with disease incidence. A few countries, such as France (DisCoVeRy) - aided by the pre-existing REACTing network - started recruiting quickly, and within four weeks, seven countries were randomizing. Unfortunately, many others were delayed by approvals from national drug regulators, ethics committees or health ministries and missed the first wave's peak. Furthermore, countries lacked clear processes for prioritizing around larger pandemic trials, with many small trials hindering progress of the global mega-trial. Over-optimistic claims that fueled off-label use of drugs, such as hydroxychloroquine, also hindered recruitment.

While both trials' results are clinically important, the global research community can learn much from examining their processes. Countries should support clinical-trial networks that can quickly activate and adapt to contribute to large, simple multi-center trials that can study both older drugs and (if not unduly over-burdened with safety monitoring) new drugs, complementing more-detailed trials by helping assess any effects on mortality. Such large, simple, core studies should be national priorities, with coordinated support from chief medical officers, healthcare providers, funders and regulators to expedite set-up processes and promote rapid recruitment.

Rapid clear answers to treatment questions have the potential to save hundreds of thousands of lives during the course of the COVID-19 pandemic. In previous pandemics, large-scale randomized trials were generally not set up in time ${ }^{8,9}$. However, RECOVERY and SOLIDARITY have set new standards and have shown that a combination of old-fashioned randomization, established clinical-trials networks and imaginative use of modern information technology can provide many rapid and reliable therapeutic answers, following the recently published rationale for pursuing the magic of randomization rather than the myth of real-world evidence ${ }^{10}$. Throughout the world, however, over-regulation and the lack of national and international efforts to facilitate appropriately large trials represent a missed opportunity to improve care. During the COVID-19 pandemic, several false claims of efficacy have emerged from non-randomized comparisons (often misleadingly referred to as 'real-world evidence'), and it has been refreshing to see how perfectly such weakly founded claims can be swept aside by evidence from properly conducted, large-scale, randomized trials.

\section{Kari A. O. Tikkinen ${ }^{1,2}$, Reza Malekzadeh ${ }^{3}$, Martin Schlegel ${ }^{4}$, Jarno Rutanen ${ }^{5}$ and Paul Glasziou ${ }^{6}{ }^{4}$}

${ }^{1}$ Departments of Urology and Public Health, University of Helsinki and Helsinki University Hospital, Helsinki, Finland. ${ }^{2}$ Department of Surgery, South Karelian Central Hospital, Lappeenranta, Finland. ${ }^{3}$ Digestive Disease Research Institute, Tehran University of Medical Sciences, Tehran, Iran. ${ }^{4}$ Department of Anesthesiology and Intensive Care,
Technical University of Munich School of Medicine, Munich, Germany. ${ }^{5}$ Department of Internal Medicine, Tampere University Hospital, Tampere, Finland. ${ }^{6}$ Institute for Evidence-Based Healthcare, Bond University, Robina, Australia.

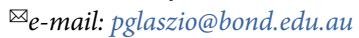

Published online: 22 September 2020 https://doi.org/10.1038/s41591-020-1077-z

References

1. Glasziou, P. P., Sanders, S. \& Hoffmann, T. Br. Med. J. 369, m1847 (2020).

2. The RECOVERY Collaborative Group. N. Engl. J. Med. https:// doi.org/10.1056/NEJMoa2021436 (2020).

3. Beigel, J.H. et al. N. Engl. J. Med. https://doi.org/10.1056/ NEJMoa2007764 (2020).

4. Yusuf, S., Collins, R. \& Peto, R. Stat. Med. 3, 409-422 (1984).

5. ISIS Collaborative Group. Lancet 2, 349-360 (1988).

6. Wise, J. \& Coombes, R. Br. Med. J. https://doi.org/10.1136/bmj. $\mathrm{m} 2670$ (2020).

7. Tenaerts, P., Madre, L. \& Landray, M. Clin. Trials 15, 5-12 (2018).

8. Muller, M. P., McGeer, A., Straus, S. E., Hawryluck, L. \& Gold, W. L. Emerg. Infect. Dis. 10, 389-394 (2004).

9. Tansey, C. M., Herridge, M. S., Heslegrave, R. J. \& Lavery, J. V. CMAJ 182, 1533-1537 (2010).

10. Collins, R., Bowman, L., Landray, M. \& Peto, R. N. Engl. J. Med. 382, 674-678 (2020).

Acknowledgements

We thank P. Horby and M. Landray (of RECOVERY), A.M. Henao-Restrepo, M.-P. Preziosi, J.-A. Røttingen and V. Sathiyamoorthy (of SOLIDARITY) and N. Peiffer-Smadja and Y. Yazdanpanah (of DisCoVeRy) for insightful advice. K.A.O.T. is supported by the Academy of Finland, Competitive Research Funding of the Helsinki and Uusimaa Hospital District and Sigrid Jusélius Foundation. The sponsors had no role in the analysis and interpretation of the data or the manuscript preparation, review, or approval.

\section{Author contributions}

K.A.O.T. and P.G. conceived of and drafted this Correspondence on the basis of interviews with trial investigators; all authors provided comments on the draft and approved the final version.

\section{Competing interests}

K.A.O.T. is the national principal investigator of the SOLIDARITY trial in Finland; R.M. is the national principal investigator of the SOLIDARITY trial in Iran; M.S. is a steering committee member of the SOLIDARITY trial in Germany; and J.R. is a steering committee member of the SOLIDARITY trial in Finland.

\section{COVID-19 puts the Sustainable Development Goals center stage}

To the Editor - A recent Comment ${ }^{1}$ and accompanying Editorial ${ }^{2}$ in Nature have raised the question of whether it is time to revise the United Nations (UN) Sustainable Development Goals (SDGs) and their various targets in the wake of the COVID-19 crisis. In their Comment, Naidoo and Fisher argue that it is "likely that many of the 169 targets will not be met by 2030 " and that "some could even be counterproductive" ${ }^{1}$. We argue for a more optimistic view and hold that the COVID-19 pandemic may help spur efforts to realize the UN's 2030 Agenda.

Rather than calling for a revision of the SDGs, COVID-19 should be seen as a catalyst for progress. COVID-19 has effectively unveiled the inequities and governance dysfunctions that the SDGs are poised to rectify ${ }^{3,4}$. By its very nature, a pandemic teaches that no one should be left behind and that no one is safe until everyone is safe.

Indeed, COVID-19 makes extant challenges impossible to ignore. It brings 